\title{
ON A THEOREM OF STEIN
}

\author{
STEVEN G. KRANTZ
}

\begin{abstract}
In this paper the Kobayashi metric on a domain in $\mathbf{C}^{n}$ is used to define a new function space. Elements of this space belong to a nonisotropic Lipschitz class. It is proved that if $f$ is holomorphic on the domain and in the classical Lipschitz space $\Lambda_{\alpha}$ then in fact $f$ is in the new function space. The result contains the original result of Stein on this subject and provides the optimal result adapted to any domain. In particular, it recovers the Hartogs extension phenomenon in the category of Lipschitz spaces.
\end{abstract}

This paper is part of a program to work out the function theory-especially the harmonic analysis-of domains in $\mathbf{C}^{n}$ in terms of invariant metrics and related constructs. The point is that, whereas in one complex dimension the principal objects of study are functions, it turns out that in several complex dimensions the principal objects of study should be domains. The correct context in which to perceive the differences between domains seems to be that of metric geometry.

The present paper is concerned with Lipschitz spaces, and draws its inspiration from [ST]. In [ST], Stein announced that holomorphic Lipschitz $\alpha$ functions on bounded $C^{2}$ domains in $\mathbf{C}^{n}, n>1$, are in fact Lipschitz $2 \alpha$ in complex tangential directions; a detailed proof appears, for instance, in [KR3]. It turns out that this result is optimal only for strongly pseudoconvex domains (see [KR6] for improved results on some other domains), and our purpose here is to find a language in which to formulate the best result for any domain. The Eisenman-Kobayashi metric and volume construction provides the most natural language. For related work using the Eisenman-Kobayashi ideas, see [KR1, KR2, KM, GK].

A second point of the present work is to eliminate the arbitrary distinction between normal and tangential directions. All directions should be treated equally, and the metric should read off the variation in smoothness.

An interesting by-product of the work is that it rediscovers the Hartogs extension phenomenon (or "Kugelsatz"-see [KR3]) in the category of Lipschitz functions. This feature is explored in $\S \S 2$ and 4.

$\S 1$ contains basic definitions and terminology, including the definition of a new Lipschitz class on domains in $\mathbf{C}^{n}$. It also contains the statement of our

Received by the editors October 12, 1988.

1980 Mathematics Subject Classification (1985 Revision). Primary 32A10; Secondary 32A30.

Work supported in part by the National Science Foundation. 
main theorem: that a holomorphic function which is Lipschitz in the classical sense is in fact in the new Lipschitz class. $\S 2$ discusses several examples. $\S 3$ contains the proof of the main theorem. $\S 4$ contains further treatment of the Kugelsatz phenomenon.

It is a pleasure to thank the referee for reading the manuscript carefully and making several helpful suggestions.

\section{Definitions, terminology, AND Statement of the theorem}

If $\Omega \subseteq \mathbf{R}^{N}$ is a bounded, connected open set (a domain), define

$\Lambda_{\alpha}(\Omega)=\{f$ continuous on $\Omega$ :

$$
\left.\sup _{\substack{x, x+h \in \Omega \\ h \neq 0}} \frac{|f(x+h)-f(x)|}{|h|^{\alpha}}+\|f\|_{L^{\infty}} \equiv\|f\|_{\Lambda_{\alpha}}<\infty\right\}
$$

$\Lambda_{1}(\Omega)=\{f$ continuous on $\Omega$ :

$$
\sup _{\substack{x, x+h, x-h \in \Omega \\ h \neq 0}} \frac{|f(x+h)+f(x-h)-2 f(x)|}{|h|}
$$

$$
\left.+\|f\|_{L^{\infty}} \equiv\|f\|_{\Lambda_{1}}<\infty\right\}
$$

and for $\alpha>1$ define $f \in \Lambda_{\alpha}(\Omega)$ if $f \in C^{1}(\Omega), f \in \Lambda_{\alpha-1}(\Omega)$, and $\nabla f \in$ $\Lambda_{\alpha-1}(\Omega)$ (with an obvious norm). See [KR4] for detailed discussion and motivation concerning these spaces. This reference also contains a thorough discussion of the finite-difference operators $\Delta_{h}^{k}$, whose definition we now recall:

If $f$ is a function on a domain $\Omega \subseteq \mathbf{R}^{N}, x \in \Omega$, and $h$ is sufficiently small then

$$
\Delta_{h}^{1} f(x) \equiv f(x+h)-f(x-h)
$$

and

$$
\Delta_{h}^{k} f(x) \equiv \Delta_{h}^{1}\left(\Delta_{h}^{k-1} f\right)(x), \quad k \geq 2 .
$$

For a continuous $f$ on a domain $\Omega \subseteq \mathbf{R}^{N}$ with $C^{2}$ boundary and $0<\alpha<k$ 
it holds that $f \in \Lambda_{\alpha}(\Omega)$ if and only if

$$
\sup _{\Omega}|f(x)|+\sup _{x, h} \frac{\left|\Delta_{h}^{k} f(x)\right|}{|h|^{\alpha}} \leq C .
$$

(Of course, the latter supremum is taken over $x, h$ such that $\Delta_{h}^{k} f(x)$ is well defined on $\Omega$.) Again, see [KR4] for a proof that this finite-difference characterization of $\Lambda_{\alpha}$ is equivalent to the original definition.

Now fix a $C^{2}$ bounded domain $\Omega \cong \mathbf{C}^{n}$. Let $B \cong \mathbf{C}^{n}$ be the unit ball and let $(\Omega, B)$ denote the collection of holomorphic maps from $B$ to $\Omega$. If $P \in \Omega$ we define the Eisenman-Kobayashi volume (see [KR1]) to be

$$
M_{K}^{\Omega}(z)=\inf \left\{\frac{1}{\left|\operatorname{det} \operatorname{Jac}_{\mathrm{C}} \Phi(0)\right|}: \Phi \in(\Omega, B), \Phi(0)=z\right\} .
$$

Once $\Omega$ is fixed and $z \in \Omega$ chosen, there is (by a normal families argument) a function $\Phi_{z} \in(\bar{\Omega}, B)$ such that

$$
M_{k}^{\Omega}(z, \xi)=1 /\left|\operatorname{det} \operatorname{Jac}_{\mathbf{C}} \Phi_{z}(0)\right| .
$$

However, $\Phi_{z}$ is not necessarily unique. Nevertheless, another normal families argument shows that the map

$$
z \rightarrow\left\|\left[\frac{\partial}{\partial z}\right]^{\beta} \Phi_{z}(0)\right\|,
$$

any multi-index $\beta$, can be taken to be upper semicontinuous on $\Omega$ (see [GRR, RE]). (Here \|\| is the standard Euclidean norm.) By Cauchy estimates, they are bounded on compact sets. In particular, we may compose these functions with curves in $B$ and integrate.

Before proceeding, we make a few trivial remarks about $M_{K}^{\Omega}$ to put its value in perspective. If $z \in \Omega$ is near $\partial \Omega$, then there is a unique nearest point $z^{\prime} \in$ $\partial \Omega$. By the unit outward normal $\nu(z)$ at $z$ we mean in fact the unit outward normal at $z^{\prime}$. Now let $z \in \Omega$ near $\partial \Omega$ and $\delta=\delta(z)=\operatorname{dist}_{\text {Euclid }}(z, \partial \Omega)$; assume for simplicity that $\mathbf{1}=(1+0 i, 0, \ldots, 0)$ is the unit outward normal $\nu(z)$ at $z$. Then the map

$$
\begin{gathered}
\Phi: B \rightarrow \Omega, \\
\Phi\left(\zeta_{1}, \ldots, \zeta_{n}\right)=z+\left(c \delta \zeta_{1}, c \sqrt{\delta} \zeta_{2}, \ldots, c \sqrt{\delta} \zeta_{n}\right),
\end{gathered}
$$

for some constant $c>0$ depending on the second fundamental form of $\partial \Omega$ near $z$, is a candidate for calculating $M_{K}^{\Omega}(z)$. In particular, notice that

$$
\left|\frac{\partial}{\partial \zeta_{1}} \Phi_{z}(0)\right|=c \delta, \quad\left|\frac{\partial}{\partial \zeta_{j}} \Phi_{z}(0)\right|=c \sqrt{\delta}, \quad j \geq 2,
$$

and therefore $M_{K}^{\Omega}(z) \leq C^{-n} \delta^{(-n-1) / 2}$. 
The following result of Royden [RO] will be useful in what follows. Let $\Omega \subset \subset \mathbf{C}^{n}$ be a domain, $P \in \partial \Omega$, and $V \subset \subset W$ neighborhoods of $P$. If $z \in V \cap \Omega$ and $\xi \in \mathbf{C}^{n}$, then

$$
F_{K}^{\Omega \cap W}(z, \xi) \leq C F_{K}^{\Omega}(z, \xi) .
$$

Here $C=C(V, W, \Omega)$. The result is proved by a normal families argument. Similar results hold for $M_{K}^{\Omega}$ and $\widetilde{M}_{K}^{\Omega}$ (which is defined below). When we use Royden's result in the sequel we call the procedure "localization."

Although our results are meaningful without the following modification to the definition of $M_{K}^{\Omega}$, we impose it to obtain the most incisive theorems.

Let $D \cong \mathbf{C}$ be the unit disc. If $z \in \Omega$ and $\xi \in \mathbf{C}^{n}$, define the infinitesimal Kobayashi metric to be

$$
F_{K}^{\Omega}(z, \xi)=\inf \left\{\frac{1}{\left\|\varphi^{\prime}(0)\right\|}: \varphi \in(\Omega, D), \varphi(0)=z, \varphi^{\prime}(0) \text { is a multiple of } \xi\right\} .
$$

Notice that $M_{K}^{\Omega}(z), F_{K}^{\Omega}(z, \xi)$ are analogous, but $F_{K}^{\Omega}$ treats one direction at $z$ at a time while $M_{K}^{\Omega}(z)$ treats all directions simultaneously. Because in many examples it is very difficult to compare the two quantities, we will mandate their comparability by replacing $M_{K}^{\Omega}(z)$ with the quantity $\widetilde{M}_{k}^{\Omega}(z)$, which we now define. Fix a tubular neighborhood $U$ of $\partial \Omega$ so that every point of $U$ has a unique nearest point in $\partial \Omega$. Then

$$
\begin{gathered}
\widetilde{M}_{K}^{\Omega}(z)=M_{k}^{\Omega}(z), \quad \text { if } z \in \Omega \backslash U ; \\
\widetilde{M}_{K}^{\Omega}(z)=\inf \left\{\frac{1}{\left|\operatorname{det} \operatorname{Jac}_{\mathbf{C}} \widetilde{\Phi}(0)\right|}: \widetilde{\Phi} \in(\Omega, B), \widetilde{\Phi}(0)=z,\right. \\
\left.\left|\left(\operatorname{Jac}_{\mathbf{C}} \widetilde{\Phi}^{-1}(z)\right)(\nu(z))\right| \leq 2 F_{K}^{\Omega}(z, \nu(z))\right\}, \quad \text { if } z \in \Omega \cap U .
\end{gathered}
$$

In short, we calculate $\widetilde{M}_{K}^{\Omega}(z)$ by restricting attention to those $\widetilde{\Phi}$ which have the property that $\left|\operatorname{Jac} \widetilde{\Phi}^{-1}\right|$ is, in the normal direction, comparable to $F_{K}^{\Omega}(z, \nu(z))$. Given a domain $\Omega$, we assume that a semicontinuous assignment

$$
z \rightarrow \widetilde{\Phi}_{z}
$$

of optimal $\widetilde{\Phi}$ 's has been selected once and for all. [Here the collection of maps $\left\{\widetilde{\Phi}_{z}\right\}$ is given the compact-open topology.]

Now we describe our new Lipschitz classes. Fix a bounded domain $\Omega \subseteq \mathbf{C}^{n}$ with $C^{2}$ boundary and associated functions $\tilde{\Phi}_{z}$. If $z \in \Omega$ let $\delta(z)$ denote the Euclidean distance of $z$ to $\partial \Omega$. Choose $\varepsilon=\varepsilon(\Omega)>0$ such that $\{z \in$ $\left.\mathbf{C}^{n}: \delta(z)<2 \varepsilon\right\}$ is a tubular neighborhood of $\partial \Omega$.

For $k=1,2, \ldots$ we define $\mathscr{C}^{k}(\Omega)=\mathscr{C}^{k}\left(\Omega, C_{0}\right)$ to be the class of all $C^{\infty}$ curves

$$
\gamma:\left[0, h_{0}\right] \rightarrow \Omega \cap\left\{z \in \mathbf{C}^{n}: \delta(z, \partial \Omega)<\varepsilon\right\}
$$


such that

$$
\left|\gamma^{\prime}(t)\right|=1, \quad \text { all } t \in\left[0, h_{0}\right]
$$

and

$$
\left|(d / d t)^{j} \gamma(t)\right| \leq C_{0} \quad \text { for all } t \in\left[0, h_{0}\right], 2 \leq j \leq k .
$$

Here $C_{0}$ is any constant. We shall later attach a number $\beta_{0}(\gamma)$, its smoothness index, to any $\gamma \in \mathscr{C}^{k}(\Omega)$. We will see in what follows that $\beta_{0}(\gamma) \geq 1$ always. Holomorphic $\Lambda_{\alpha}$ functions on $\Omega$ will in general turn out to be Lipschitz of order $\beta_{0}(\gamma) \cdot \alpha$ along a curve $\gamma$. The Lipschitz norm will depend on $C_{0}$.

Observe that $\mathscr{C}^{k}$ should not be confused with the classical function space of $k$ times continuously differentiable functions. We use the notation $\mathscr{C}^{k}$ to preserve some consistency with [KR5, KR6, ST].

The following notation will make future arguments easier to read: once a domain $\Omega$ and a curve $\gamma \in \mathscr{C}^{k}(\Omega)$ are fixed, then

$$
D_{N} \widetilde{\boldsymbol{\Phi}}_{\gamma(t)} \equiv 1 /\left\|\left[\mathrm{Jac}_{\mathbf{C}}\left(\widetilde{\boldsymbol{\Phi}}_{\gamma(t)}^{-1}\right)\right] \nu(\gamma(t))\right\|
$$

and

$$
D_{T} \widetilde{\Phi}_{\gamma(t)} \equiv 1 /\left\|\left[\mathrm{Jac}_{\mathbf{C}}\left(\widetilde{\Phi}_{\gamma(t)}\right)\right]^{-1} \gamma^{\prime}(t)\right\|
$$

When $\gamma$ is complex normal at $t$, then of course $D_{N} \widetilde{\Phi}_{\gamma(t)}=D_{T} \widetilde{\Phi}_{\gamma(t)}$. Notice, for instance, that with this new notation the condition on $\Phi$ in the definition of $\widetilde{M}_{K}^{\Omega}(z)$ is that $\left|D_{N} \widetilde{\Phi}_{\gamma(t)}\right|^{-1} \leq 2 F_{K}^{\Omega}(\gamma(t), \nu(\gamma(t)))$.

Let $\nu: \bar{\Omega} \rightarrow \mathbf{C}^{n}$ be a $C^{1}$ function which satisfies the conditions

$$
\begin{aligned}
& \text { for points } z \in \bar{\Omega} \cap\left\{z \in \mathbf{C}^{n}: \delta(z, \partial \Omega)<2 \varepsilon\right\}, \\
& \nu(z) \text { is the (well-defined) outward unit normal at } z .
\end{aligned}
$$

For any curve $\gamma \in \mathscr{C}^{1}$ define

$$
\lambda_{0}(\gamma)=\sup _{t \in\left[0, h_{0}\right]} \log _{\delta(\gamma(t))}\left|D_{N} \widetilde{\Phi}_{\gamma(t)}\right| .
$$

By the remarks following the definition of $M, \widetilde{M}$, it follows that

$$
\frac{1}{2} \leq \lambda_{0}(\gamma) \leq 1
$$

For a given curve $\gamma$ near $\partial \Omega$ and $0<\mu$ small we define

$$
\gamma_{\mu}(t)=\gamma(t)-\mu \cdot \nu(\gamma(t)) .
$$

When we study the Lipschitz smoothness of a function $f$ along a curve $\gamma \in \mathscr{C}^{k}$, we need only consider $\Delta_{h}^{k}(f \circ \gamma)$ for $h$ small. Thus we restrict attention to $0<h<\varepsilon=\varepsilon(\Omega)$. For $\gamma \in \mathscr{C}^{k}(\Omega)$ and $h$ fixed, define

$$
\sigma_{\beta}(t)=\log _{\left[D_{N} \widetilde{\Phi}_{h^{\beta}(t)}\right]}\left[D_{T} \widetilde{\Phi}_{\gamma_{h^{\beta}}(t)}\right]
$$


and

$$
\varphi(\beta)=\frac{1}{\beta}-1-\lambda_{0}(\gamma) \cdot\left[\frac{1}{h} \int_{0}^{h} \sigma_{\beta}(t) d t-1\right], \quad 1 \leq \beta<\infty .
$$

Observe that, by the remarks following the definition of the Kobayashi metric,

$$
0<\sigma_{\beta}(t) \leq 1, \quad \text { all } t \in\left[0, h_{0}\right] .
$$

It follows that

$$
\varphi(1) \geq 0
$$

and, for $\beta$ large enough, $\varphi(\beta)<0$. [Notice here that some definition chasing and use of the estimate (1) implies that $\sigma_{\beta}(t)$ is bounded from 0 .] We select

$$
\beta_{0}(\gamma)=\inf \{\beta \in[1, \infty): \varphi(\beta)<0\} .
$$

When no confusion is possible we write $\lambda_{0}=\lambda_{0}(\gamma), \beta_{0}=\beta_{0}(\gamma)$.

Definition. Let $0<\alpha<\infty$. A continuous function

$$
f: \Omega \rightarrow \mathbf{C}
$$

is said to be in the space $\mathscr{L}_{\alpha}(\Omega)$ if

(i) for any $\gamma \in \mathscr{C}^{k}(\Omega), t \in\left(0, h_{0}\right), h$ sufficiently small, and $k$ sufficiently large, it holds that

$$
\left|\Delta_{h}^{k}(f \circ \gamma(t))\right| \leq C \cdot|h|^{\beta_{0} \lambda_{0}(\alpha-1)+\beta_{0}} .
$$

(Here $k>\beta_{0} \lambda_{0}(\alpha-1)+\beta,|h|<\left(\min \left\{|t|,\left|h_{0}-t\right|\right\}\right) / 2 k$ will do. $)$

(ii) If $\gamma:[0,1] \rightarrow \Omega$ satisfies $\left|\gamma^{\prime}(t)\right| \equiv 1,\left|(d / d t)^{j} \gamma(t)\right| \leq C_{0}, 2 \leq j \leq k$, $t \in\left[0, h_{0}\right]$, then

$$
\left|\Delta_{h}^{k} f \circ \gamma(t)\right| \leq C|h|^{\alpha} .
$$

Our main theorem is

Theorem. If $\Omega \subseteq \mathbf{C}^{n}$ is a bounded domain with $C^{2}$ boundary and $f: \Omega \rightarrow \mathbf{C}$ is holomorphic and in $\Lambda_{\alpha}(\Omega)$ then $f \in \mathscr{L}_{\alpha}(\Omega)$.

Remark. Our definition of $\mathscr{L}_{\alpha}$ is somewhat indirect. However, this is necessary to avoid the intractable calculation of the Kobayashi metric on general domains. As the examples of the next section will show, most of the interesting cases satisfy $\lambda_{0}=1$ (asymptotically, as the distance of $\gamma$ to $\partial \Omega$ tends to 0 ); hence the critical part (i) of the definition simplifies to

$$
\left|\Delta_{h}^{k}(f \circ \gamma(t))\right| \leq C|h|^{\beta_{0} \alpha} .
$$

Thus $\beta_{0}(\gamma)$ is the smoothness index to which we alluded earlier. The more complicated formulation (i) of the definition allows us to rediscover the Hartogs extension phenomenon in the Lipschitz category. See the exposition of this matter in the next section.

Although in the end the examples and the theorems vindicate the tedious definitions we have presented, perhaps a few additional words of explanation 
are in order now . The papers [ST] and [KR5, KR6] have already established the naturality of increased smoothness in tangential directions displayed in (2). What motivates the definition of $\beta_{0}$ ? In [ST] (see details in [KR3]), the crucial geometric fact is this: at a point $P$ near $\partial \Omega$ the domain $\Omega$ contains a right (i.e., affine) analytic disc of radius $\delta(P)$ and center $P$ with orientation in the normal direction; however, if the orientation is in the tangential direction, the disc may be taken to have radius $\delta(P)^{1 / 2}$, and the smoothness index in Stein's theorem is the quotient of the exponents of $\delta(P)$ which occur here; $2=1 /(1 / 2)$. It is the same act at every boundary point. The point of view of the present paper is that it is more natural to use embedded discs (i.e., analytic discs in the usual sense of several complex variables-see [KR3]). One still obtains the smoothness index by measuring the size of the discs as powers of $\delta(P)$ and calculating the ratio of the exponents: this accounts for the logarithmic expressions used in the definitions of $\lambda_{0}$ and $\sigma_{\beta}$. The average that is used to define $\varphi$ and the indirect definition of $\beta_{0}$ are ways of dealing with the facts that (i) the relevant quantities vary from point to point and (ii) it is in general impossible to calculate the quantity $\widetilde{M}_{K}^{\Omega}$.

\section{EXAMPLES}

Example 1. If $\Omega=D=\{z \in \mathbf{C}:|z|<1\}$, then

$$
\widetilde{M}_{k}^{\Omega}(z)=\frac{1}{1-|z|^{2}} \quad \text { and } \quad \widetilde{\Phi}_{z}(\zeta)=\frac{z+\zeta}{1+\bar{z} \zeta}, \quad \widetilde{\Phi}_{z}^{\prime}(0)=1-|z|^{2} .
$$

Here and throughout, \|\| is Euclidean vector length. If $\gamma \in \mathscr{C}^{k}(D)$ then $\left\|\gamma^{\prime}(t)\right\|=1$ for all $t$ and

$$
\lambda_{0}(\gamma)=\sup _{t}\left|\log _{(1-\|\gamma(t)\|)}\left(1 /\left(1-\|\gamma(t)\|^{2}\right)\right)\right| \sim 1 .
$$

Here we use the notation $\alpha \sim \beta$ to mean that $\alpha / \beta \rightarrow 1$ as the Euclidean distance to $\partial \Omega$ of either the point or the curve in question tends to 0 (context will make clear which is meant). Similarly,

$$
\sigma_{\beta}(t)=1 \text {. }
$$

It follows that $\varphi(\beta)=1 / \beta-1$ and $\beta_{0}(\gamma)=1$. Thus a holomorphic $f \in \Lambda_{\alpha}(D)$ satisfies

$$
f \circ \gamma \in A_{\beta_{0} \lambda_{0}(\alpha-1)+\beta_{0}}\left(\left[0, h_{0}\right]\right),
$$

where $\beta_{0} \lambda_{0}(\alpha-1)+\beta_{0} \sim \alpha$. In other words, $D \subseteq \mathbf{C}^{1}$ has no distinguished directions: there is no "automatic increase in smoothness" for a holomorphic Lipschitz function on the disc. 
Example 2. Let $\Omega=B=\left\{z \in \mathbf{C}^{2}:\|z\|<1\right\}$. Fix $z \in B$. Then it is known that

$$
\begin{gathered}
\widetilde{M}_{k}^{\Omega}(z)=1 /\left(1-\|z\|^{2}\right)^{3 / 2} \\
\widetilde{\Phi}(\zeta)=\left[\frac{z_{1}}{\|z\|} \frac{\|z\|+\zeta_{1}}{1+\|z\| \zeta_{1}}-\frac{\overline{z_{2}}}{\|z\|} \frac{\sqrt{1-\|z\|^{2}} \zeta_{2}}{1+\|z\| \zeta_{1}},\right. \\
\left.\frac{z_{2}}{\|z\| z \|+\zeta_{1}} \frac{\overline{z_{1}}}{1+\|z\| \zeta_{1}} \frac{\sqrt{1-\|z\|^{2}} \zeta_{2}}{1+\|z\| \zeta_{1}}\right], \\
\operatorname{Jac}_{\mathbf{C}} \widetilde{\Phi}_{z}(0)=\left[\begin{array}{cc}
\frac{z_{1}}{\|z\|}\left(1-\|z\|^{2}\right) & -\frac{\bar{z}}{\|z\|} \sqrt{1-\|z\|^{2}} \\
\frac{z_{2}}{\|z\|}\left(1-\|z\|^{2}\right) & \frac{\frac{1}{z_{1}}}{\|z\|} \sqrt{1-\|z\|^{2}}
\end{array}\right], \\
\left|\operatorname{det} \operatorname{Jac}_{\mathbf{C}} \widetilde{\Phi}_{z}(0)\right|=\left(1-\|z\|^{2}\right)^{3 / 2} \\
\left|D_{N} \widetilde{\Phi}_{z}\right|=\left(1-\|z\|^{2}\right),
\end{gathered}
$$

and if $\gamma \in \mathscr{C}^{k}$ is a complex tangential curve,

$$
\left|D_{T} \widetilde{\Phi}_{\gamma(t)}\right|=\sqrt{1-\|\gamma(t)\|^{2}}
$$

while if $\gamma \in \mathscr{C}^{k}$ complex normal,

$$
\left|D_{T} \widetilde{\boldsymbol{\Phi}}_{\gamma(t)}\right|=1-\|\gamma(t)\|^{2} .
$$

Thus, for a complex tangential curve,

$$
\begin{gathered}
\lambda_{0}(\gamma) \sim 1, \quad \sigma_{\beta} \sim \frac{1}{2}, \\
\varphi(\beta) \sim 1 / \beta-1-1 \cdot\left[-\frac{1}{2}\right]=1 / \beta-\frac{1}{2} .
\end{gathered}
$$

Thus $\beta_{0} \sim 2$ and a holomorphic $\Lambda_{\alpha}$ function is $\Lambda_{2 \alpha}$ along $\gamma$. For a complex normal curve,

$$
\begin{aligned}
& \lambda_{0}(\gamma) \sim 1, \quad \sigma_{\beta} \sim 1, \\
& \varphi(\beta) \sim 1 / \beta-1[1-1],
\end{aligned}
$$

so $\beta_{0} \sim 1$ and $f$ is $\Lambda_{\alpha}$ along $\gamma$. There is no increased smoothness in normal directions, as expected.

Example 3. In case $\Omega \subset \subset \mathbf{C}^{n}$ is smooth and strongly pseudoconvex, the asymptotic boundary behavior of the Kobayashi metric is precisely the same as that on the ball (see [GR, GK]). So one obtains that a holomorphic $\Lambda_{\alpha}$ function is $\Lambda_{2 \alpha}$ in complex tangential directions, but only $\Lambda_{\alpha}$ in complex normal directions.

Example 4. In case

$$
\Omega=\Omega_{m}=\left\{z \in \mathbf{C}^{2}:\left|z_{1}\right|^{2}+\left|z_{2}\right|^{2 m}<1\right\}
$$


and $\gamma$ is essentially a complex tangential curve near the weakly pseudoconvex point $(1,0)$ - say $\gamma(t)=(1-\delta, t)$ for some fixed $\delta>0$ and $0 \leq t \leq \delta^{1 / 2 m}$ then it can be calculated (using [CA], for instance) that

$$
D_{T} \widetilde{\Phi}_{\gamma(t)} \sim C \cdot \delta(\gamma(t))^{1 / 2 m} .
$$

Also

$$
D_{N} \widetilde{\Phi}_{\gamma(t)} \sim C \cdot \delta(\gamma(t))
$$

Therefore

$$
\lambda_{0}(\gamma) \sim 1 \text { and } \sigma_{\beta}(t) \sim 1 / 2 m
$$

We conclude that

$$
\varphi(\beta) \sim 1 / \beta-1-1 \cdot[1 / 2 m-1]
$$

and $\beta_{0} \sim 2 m$. Therefore for a holomorphic $f \in \Lambda_{\alpha}(\Omega)$ we have that $f \circ \gamma \in$ $\Lambda_{2 m \alpha}([0, h])$.

Example 5. If $\Omega$ is a domain of finite type in $\mathbf{C}^{2}$, then the estimates of Catlin [CA] can be used to calculate the increased tangential smoothness of holomorphic Lipschitz functions. Roughly speaking, one finds that near a point of type $m$ the smoothness of a Lipschitz $\alpha$ function is increased by a factor of $m+1-$ that is, $f$ is Lipschitz of order $(m+1) \alpha$ along complex tangential curves. The details of these calculations, together with applications to estimates for the $\bar{\partial}$ Neumann problem, will appear in [CK].

Example 6. Let

$$
\mathbf{\Omega}=\left\{z \in \mathbf{C}^{2}:|z|<2,|z-(1,0)|>1\right\}
$$

and let $\gamma \in \mathscr{C}(\Omega)$ be a complex normal curve near the boundary point $(1,0)$, say that $\gamma(t)=(1-\delta-t, 0), 0 \leq t \leq h_{0}$, and $\delta>0$ fixed. Then it turns out that

$$
D_{N} \widetilde{\Phi}_{\gamma(t)} \sim C \cdot(\delta+t)^{-3 / 4} \text {. }
$$

For our purposes, it suffices to see that

$$
D_{N} \widetilde{\Phi}_{\gamma(t)} \geq C \cdot(\delta+t)^{3 / 4}
$$

and this is demonstrated by the map

$$
\begin{gathered}
\varphi: B \rightarrow \Omega, \\
\left(\zeta_{1}, \zeta_{2}\right) \rightarrow\left(1-\delta-t+(\delta+t)^{3 / 4} \zeta_{1} / 50,8 \zeta_{1}^{2}+\delta^{1 / 2} \zeta_{2} / 15\right) .{ }^{1}
\end{gathered}
$$

It follows from $(*)$ that

$$
\lambda_{0}(\gamma) \leq \frac{3}{4}
$$

Also

$$
\sigma_{\beta}(t)=1, \quad \text { all } \beta \geq 1 .
$$

'The opposite inequality is derived from Schwarz's lemma. These facts were discovered by the author and Erik Low in unpublished work. They were also known to Halsey Royden, N. Sibony, and others. See [KR7]. 
Therefore

$$
\gamma(\beta)=1 / \beta-1
$$

and $\beta_{0}=1$. Now this is the example in which we must use the full force of part (i) of the definition of $\mathscr{L}_{\alpha}$; for now $\lambda_{0}(\gamma) \neq 1$. We conclude that a holomorphic $f \in \Lambda_{\alpha}(\Omega)$ satisfies $f \circ \gamma \in \Lambda_{3 \alpha / 4+1 / 4}$. For $0<\alpha<1$, this shows that even in complex normal directions there is an increase in smoothness. It follows from results in [KR5] that we have

( $f$ holomorphic and in $\Lambda_{\alpha}(\Omega)$ )

$$
\Rightarrow\left(f \in \Lambda_{3 \alpha / 4+1 / 4}(\overline{\Omega \cap B(1,1)}) \text { in all directions }\right) .
$$

For $0<\alpha<1$, this is a version of the Hartogs extension phenomen in the Lipschitz category: on this domain $\Omega$ a holomorphic $f \in \Lambda_{\alpha}(\Omega)$ is automatically smoother near $(1,0)$. In $\S 4$ we show how to modify the argument to obtain that $f \in \Lambda_{\alpha}(\Omega)$ implies $f \in C^{\infty}(\overline{\Omega \cap B(0,1)})$.

It is worth noting that the Kobayashi metric estimates described here and the subsequent $C^{\infty}$ version of the Hartog phenomenon hold near any boundary point at which the Levi form has a negative eigenvalue. See [KR7].

\section{Proof of THE THEOREM}

In Stein's work, the key fact (for small $\alpha$ ) is that, for a holomorphic Lipschitz $\alpha$ function $f$,

$$
|\nabla f(z)| \leq C \delta(t)^{\alpha-1},
$$

while for a tangential derivative $D_{\tau}$ it holds that

$$
\left|D_{\tau} f(z)\right| \leq C \delta(z)^{\alpha-1 / 2} .
$$

For us it is more convenient to formulate these matters differently.

Lemma 1. If $f$ is a holomorphic $\Lambda_{\alpha}$ function on $\Omega$ and $(\partial / \partial \zeta)^{\beta}$ is any differential monomial then

$$
\left|(\partial / \partial \zeta)^{\beta}\left(f \circ \widetilde{\Phi}_{z}\right)(0)\right| \leq C \cdot\left|D_{N} \widetilde{\Phi}_{z}(0)\right|^{\alpha} .
$$

Remark. It is a bit surprising that the constant on the right is independent of $\alpha$. But examination of the chain rule on the left alleviates the surprise.

Proof of lemma. This is really a reformulation of Stein's basic estimates, as presented, for instance, in [KR3]. We outline the proof.

First we treat the case of $0<\alpha<1$. Assume without loss of generality that $N_{z}=(1+i 0,0, \ldots 0)$. Let $\varphi \in C_{c}^{\infty}\left(D\left(0, \frac{1}{2}\right)\right)$ satisfy $\varphi \geq 0, \varphi$ radial, $\int \varphi(z) d A=1$, where $d A$ is two-dimensional area. Write elements of $B$ as $\zeta=\left(\zeta_{1}, \ldots, \zeta_{n}\right)$ and $\zeta_{j}=\rho_{j}+i \eta_{j}$. Then, by the mean value property of holomorphic functions,

$$
\begin{aligned}
\left|\frac{\partial}{\partial \rho_{1}} f \circ \widetilde{\boldsymbol{\Phi}}_{z}(\zeta)\right| & =\left|\frac{\partial}{\partial \rho_{1}} \iint f \circ \widetilde{\boldsymbol{\Phi}}\left(\xi_{1}, \zeta_{2}, \ldots, \zeta_{n}\right) \varphi\left(\zeta_{1}-\xi_{1}\right) d A\left(\xi_{1}\right)\right| \\
& =\left|\iint f \circ \widetilde{\boldsymbol{\Phi}}_{z}\left(\xi_{1}, \zeta_{2}, \ldots, \zeta_{n}\right) \frac{\partial}{\partial \rho_{1}} \varphi\left(\zeta_{1}-\xi_{1}\right) d A\left(\xi_{1}\right)\right| .
\end{aligned}
$$


Since $\left(\partial / \partial \rho_{1}\right) \varphi\left(\zeta_{1}-\xi_{1}\right)$ has mean value zero, this last equals

$$
\begin{aligned}
&=\mid \iint\left[f \circ \widetilde{\Phi}_{z}\left(\xi_{1}, \zeta_{2}, \ldots, \zeta_{n}\right)-f \circ \widetilde{\Phi}_{z}\left(0, \zeta_{2}, \ldots, \zeta_{n}\right)\right] \times \frac{\partial}{\partial \rho_{1}} \varphi\left(\zeta_{1}-\xi_{1}\right) d A\left(\xi_{1}\right) \mid \\
& \leq C \iint\left|\widetilde{\Phi}_{z}\left(\xi_{1}, \zeta_{2}, \ldots, \zeta_{n}\right)-\widetilde{\Phi}_{z}\left(0, \zeta_{2}, \ldots, \zeta_{n}\right)\right|^{\alpha}\left|\frac{\partial}{\partial \rho_{1}} \varphi\left(\zeta_{1}-\xi_{1}\right)\right| d A\left(\xi_{1}\right)
\end{aligned}
$$

since $f \in \Lambda_{\alpha}(\Omega)$. Take $\zeta=0$. Since we normalized $N_{z}$ to be $\left(1+0_{i}, 0\right.$, $\ldots, 0)$, this last is

$$
\leq C \iint\left|\xi_{1}\right|^{\alpha}\left|D_{N} \widetilde{\Phi}_{z}\left(\xi_{1}^{\prime}, 0, \ldots, 0\right)\right|^{\alpha}\left|\frac{\partial}{\partial \rho_{1}} \varphi\left(\zeta_{1}-\xi_{1}\right) d A\left(\xi_{1}\right)\right|,
$$

some $\xi_{1}^{\prime}=\xi_{1}^{\prime}\left(\xi_{1}, 0, \ldots, 0\right)$,

$$
=C \iint\left|\xi_{1}\right|^{\alpha}\left|D_{N}\left(\widetilde{\Phi}_{z} \circ\left(m_{\xi_{1}}^{\prime}\right)^{-1} \circ m_{\xi_{1}^{\prime}}\right)\left(\xi_{1}^{\prime}, 0, \ldots, 0\right)\right|^{\alpha} d A\left(\xi_{1}\right),
$$

where $m_{\xi_{1}^{\prime}}$ is a Möbius transformation that moves $\left(\xi_{1}^{\prime}, 0,0, \ldots, 0\right)$ to $(0, \ldots$, $0)$. We take

$$
m_{\xi_{1}^{\prime}}\left(w_{1}, \ldots, w_{n}\right)=\left[\frac{w_{1}-\xi_{1}^{\prime}}{1-\bar{\xi}_{1}^{\prime} w_{1}}, \frac{\sqrt{1-\left|\xi_{1}\right|^{2}} w_{2}}{1-\bar{\xi}_{1}^{\prime} w_{1}}, \ldots, \frac{\sqrt{1-\left|\xi_{1}\right|^{2}} w_{n}}{1-\bar{\xi}_{1}^{\prime} w_{1}}\right] .
$$

Then

$$
\mathrm{Jac}_{\mathbf{C}} m_{\xi_{1}^{\prime}}\left(\xi_{1}^{\prime}, 0, \ldots, 0\right)=\left[\begin{array}{ccccc}
\frac{1}{1-\left|\xi_{1}^{\prime}\right|^{2}} & 0 & \cdots & & 0 \\
0 & \frac{1}{\sqrt{1-\left|\xi_{1}^{\prime}\right|^{2}}} & 0 & \cdots & 0 \\
0 & 0 & \frac{1}{\sqrt{1-\left|\xi_{1}^{\prime}\right|^{2}}} & & 0 \\
0 & 0 & 0 & \cdots & \frac{1}{\sqrt{1-\left|\xi_{1}^{\prime}\right|^{2}}}
\end{array}\right]
$$

and

$$
\operatorname{det} \operatorname{Jac}_{\mathbf{C}} m_{1}^{\prime}\left(\xi_{1}^{\prime}, 0, \ldots, 0\right)=\left[\frac{1}{1-\left|\xi_{1}^{\prime}\right|^{2}}\right]^{1+(n-1) / 2}>1 .
$$

This calculation, together with the maximality of $\widetilde{\Phi}_{z}$, gives that

$$
(*) \leq C\left|D_{N} \widetilde{\Phi}_{z}(0)\right|^{\alpha} .
$$

This is the desired estimate for $\partial / \partial \zeta_{1} \approx D_{N}$.

Now let us estimate

$$
\frac{\partial}{\partial \zeta_{2}} \frac{\partial}{\partial \zeta_{1}}\left(f \circ \widetilde{\Phi}_{z}\right)(\zeta)=\frac{\partial}{\partial \zeta_{2}} \frac{\partial}{\partial \zeta_{1}} \int f \circ \widetilde{\Phi}_{z}\left(\zeta_{1}, \xi_{2}, \zeta_{3}, \ldots, \zeta_{n}\right) \varphi\left(\zeta_{2}-\xi_{2}\right) d A\left(\xi_{2}\right)
$$


where $\varphi$ is the nonnegative radial function of unit mass that we used before. This last equals

$$
\int \frac{\partial}{\partial \zeta_{1}}\left(f \circ \widetilde{\Phi}_{z}\right)\left(\zeta_{1}, \xi_{2}, \zeta_{3}, \ldots, \zeta_{n}\right) \frac{\partial}{\partial \zeta_{2}} \varphi\left(\zeta_{2}-\xi_{2}\right) d A\left(\xi_{2}\right) .
$$

Setting $\zeta=0$ and taking absolute values gives

$$
\left|\frac{\partial}{\partial \zeta_{2}} \frac{\partial}{\partial \zeta_{1}}\left(f \circ \widetilde{\boldsymbol{\Phi}}_{z}\right)(0)\right| \leq C \int\left|\frac{\partial}{\partial \zeta_{1}}\left(f \circ \widetilde{\boldsymbol{\Phi}}_{z}\right)\left(0, \xi_{2}, 0, \ldots, 0\right)\right|^{\alpha} d A\left(\xi_{2}\right) .
$$

But using a Möbius transformation as before allows us to estimate this by

$$
C\left|\frac{\partial}{\partial \zeta_{1}}\left(f \circ \widetilde{\Phi}_{z}\right)(0)\right|^{\alpha}=C\left|D_{N}\left(f \circ \widetilde{\Phi}_{z}\right)(0)\right|^{\alpha},
$$

which we know to be

$$
\leq C\left|D_{N} \widetilde{\Phi}_{z}(0)\right|^{\alpha}
$$

We now have

$$
\left|\frac{\partial}{\partial \zeta_{2}} \frac{\partial}{\partial \zeta_{1}}(f \circ \widetilde{\Phi})(0)\right| \leq C\left|D_{N} \widetilde{\Phi}_{z}(0)\right|^{\alpha} \text {. }
$$

In fact, the same estimate obtains with $0=(0,0)$ on the left replaced by $\left(\zeta_{1}, 0\right)$ with $\left|\zeta_{1}\right| \leq \frac{1}{4}$. Integrating out the $\zeta_{1}$ variable gives

$$
\left|\frac{\partial}{\partial \zeta_{2}}(f \circ \widetilde{\Phi})(0)\right| \leq C\left|D_{N} \widetilde{\Phi}_{z}(0)\right|^{\alpha} .
$$

Higher derivatives of $f \circ \Phi_{z}$ are estimated similarly using standard techniques, for which see [KR3] or [KR5].

Lemma 2. Let $\Omega \subseteq \mathbf{C}^{n}$ be a domain with $C^{2}$ boundary. Let $P \in \partial \Omega$ and let $V$ be a small open neighborhood of $P$. Let $z \in V$ be within the tubular neighborhood $U$ of $\partial \Omega$. Then, for $\eta>0$ sufficiently small,

$$
\log _{\delta(z)}\left|D_{N} \widetilde{\Phi}_{z}\right| \leq C \log _{\delta(z-\eta \nu(z))}\left|D_{N} \widetilde{\Phi}_{z-\eta \nu(z)}\right| .
$$

I he constant $C \rightarrow 1$ as $\delta(z) \rightarrow 0$ and $\eta \rightarrow 0$ independently.

Proof. This is essentially a scaling argument. Fix neighborhoods $W^{\prime}$ $V$ of $P$. We use Royden's localization procedure. Assume without loss of generality that $\nu(z)=(1+0 i, 0, \ldots, 0)$. Let $\varepsilon>0$. Let $\widetilde{\Phi} \in(\Omega \cap W \cap U$, $B)$ be a map such that $1 /\left|D_{N} \widetilde{\Phi}_{z}\right| \leq 2 F_{k}^{\Omega}(z, \nu(z))$ and

$$
1 /|\operatorname{det} \widetilde{\Phi}(0)| \leq M_{k}^{\Omega}(z)+\varepsilon \text {. }
$$

Assume for simplicity that $n=2$. Then

$$
\hat{\Phi}\left(\zeta_{1}, \zeta_{2}\right)=\left(z_{1}+c_{1} \zeta+\cdots, z_{2}+d_{2} \zeta^{2}+\cdots\right) .
$$

Define

$$
\widetilde{\boldsymbol{\Phi}}^{\eta}\left(\zeta_{1}, \zeta_{2}\right)=\left(z_{1}-\eta \nu(z)+\frac{1}{\left|c_{1}\right|}(\delta(z)+\eta)^{\log _{\delta(z)}\left|c_{1}\right|}\left(c_{1} \zeta+\cdots\right), \mu\left(d_{2} \eta^{2}+\cdots\right)\right),
$$


where $\mu$ is a constant to be selected. If $W, W^{\prime}$ are small enough, and if $\mu$ is chosen appropriately (depending on the second fundamental form of $\partial \Omega$ near $P)$, then $\widetilde{\Phi}^{\eta} \in\left(\Omega \cap W^{\prime} \cap U, B\right)$ and $\widetilde{\Phi}^{\eta}(0)=z-\eta \nu(z)$. Thus $\widetilde{\Phi}^{\eta}$ is a candidate for $\widetilde{\Phi}_{z-\eta \nu(z)}$. It follows that

$$
\begin{gathered}
\log _{\delta(z)+\eta}\left|D_{N} \tilde{\Phi}_{z}\right| \geq \log _{\delta(z)+\eta}\left|C \cdot(\delta(z)+\eta)^{\log _{\delta(z)}\left|c_{1}\right|}\right| \\
=\log _{\delta(z)}\left|\frac{\partial}{\partial \zeta_{1}} \widetilde{\Phi}(0)\right|+\log _{(\delta(z)+\eta)} C .
\end{gathered}
$$

Of course, $\log _{\delta(z)+\eta} C \sim 0$ when $\eta(z), \delta$ are small so it is negligible (the first term, by comparison, is always $\geq \frac{1}{2}$ ). By the choice of $\widetilde{\Phi}$, the result follows.

We now turn to the proof of the theorem. Fix a holomorphic $f$ in $\Lambda_{\alpha}(\Omega)$. Let $\gamma \in \mathscr{C}^{k}(\Omega)$, with domain $\left[0, h_{0}\right]$. Fix $k$ large. Choose $\beta_{0}(\gamma)$ as in $\S 1$. Fix a positive $h$ which is smaller than $h_{0} / 2 k$ and smaller than the $\varepsilon>0$ used to define the tubular neighborhood $U$. Define auxiliary curves

$$
\begin{array}{ll}
\gamma_{1}(t)=\gamma(0)-t \nu(\gamma(0)), & 0 \leq t \leq h^{\beta_{0}}, \\
\gamma_{2}(t)=\gamma(t)-h^{\beta_{0}} \nu(\gamma(0)), & 0 \leq t \leq h, \\
\gamma_{3}(t)=\gamma(0)+\left(h^{\beta_{0}}-t\right) \nu(\gamma(h)), & 0 \leq t \leq h^{\beta_{0}} .
\end{array}
$$

Assume at first that $0<\alpha<\min \left\{\left(1-\beta_{0}+\beta_{0} \lambda_{0}\right) / \beta_{0} \lambda_{0}, \operatorname{avg}_{\left[0, h_{0}\right]}\left(\sigma_{\beta_{0}}\right)\right\}$. We comment on larger $\alpha$ later. Then

$$
\begin{aligned}
&|f(\gamma(h))-f(\gamma(0))| \leq\left|f\left(\gamma_{1}(0)\right)-f\left(\gamma_{1}\left(h^{\beta_{0}}\right)\right)\right| \\
&+\left|f\left(\gamma_{2}(0)\right)-f\left(\gamma_{2}(h)\right)\right| \\
&+\left|f\left(\gamma_{3}(0)\right)-f\left(\gamma_{3}\left(h^{\beta_{0}}\right)\right)\right| \\
& \equiv \mathrm{I}+\mathrm{II}+\mathrm{III} .
\end{aligned}
$$

To estimate II, we write

$$
\left|f\left(\gamma_{2}(0)\right)-f\left(\gamma_{2}(h)\right)\right|=\left|\int_{0}^{h}\left(f \circ \gamma_{2}\right)^{\prime}(t) d t\right| .
$$

Now for each $t$ we have

$$
\begin{aligned}
\left|\left(f \circ \gamma_{2}\right)^{\prime}(t)\right| & =\left|\nabla f\left(\gamma_{2}(t)\right) \cdot \gamma_{2}^{\prime}(t)\right| \\
& =\left|\nabla f\left(\gamma_{2}(t)\right) \cdot \nabla \widetilde{\Phi}_{\gamma_{2}(t)}(0)\left(\nabla \widetilde{\Phi}_{\gamma_{2}(t)}(0)\right)^{-1} \cdot \gamma_{2}^{\prime}(t)\right| \\
& \leq\left|\left(\nabla\left(f \circ \widetilde{\Phi}_{\gamma_{2}(t)}\right)(0)\right)\right| \cdot\left|D_{T(t)}\left(\widetilde{\Phi}_{\gamma_{2}(t)}\right)\right|^{-1},
\end{aligned}
$$

where $T(t)$ is the unit tangent vector $\gamma^{\prime}(t)$. By Lemma 1, the last line is

$$
\begin{aligned}
& \leq\left|D_{N} \widetilde{\Phi}_{\gamma_{2}(t)}(0)\right|^{\alpha} \cdot\left|D_{T(t)} \widetilde{\Phi}_{\gamma_{2}(t)}(0)\right|^{-1} \\
& \leq\left|D_{N} \widetilde{\Phi}_{\gamma_{2}(t)}(0)\right|^{\alpha-\sigma_{\beta_{0}}(t)}
\end{aligned}
$$


where Lemma 2 has been used. Thus, from $(*)$ and $(* *)$, we have

$$
\begin{aligned}
\left|f\left(\gamma_{2}(0)\right)-f\left(\gamma_{2}(h)\right)\right| & \leq \int_{0}^{h}\left|D_{N} \widetilde{\Phi}_{\gamma_{2}(t)}\right|^{\alpha-\sigma_{\beta_{0}}(t)} d t \\
& \leq \int_{0}^{h} \mu_{0}^{\alpha-\sigma_{\beta_{0}(t)}} d t,
\end{aligned}
$$

where $\mu_{0}=\inf _{t}\left|D_{N} \widetilde{\Phi}_{\gamma_{2}(t)}\right|$. This last is

$$
\leq h \cdot \mu_{0}^{\left[\int_{0}^{h} \alpha-\sigma_{\beta_{0}}(t) d t\right]}=h \cdot \mu_{0}^{\left(\alpha-\operatorname{avg}\left(\sigma_{\beta_{0}}\right)\right)}
$$

by Jensen's inequality. However $\alpha-\operatorname{avg}\left(\sigma_{\beta_{0}}\right)<0$ so

$$
\begin{aligned}
\mu_{0}^{\alpha-\operatorname{avg}\left(\sigma_{\beta_{0}}\right)} & \leq \inf _{t}\left|D_{N} \widetilde{\Phi}_{\gamma_{2}(t)}\right|^{\alpha-\operatorname{avg}\left(\sigma_{\beta}\right)} \\
& =\left|\inf _{t} \delta\left(\gamma_{2}(t)\right)^{\log _{\delta\left(y_{2}(t)\right)}\left|D_{N} \widetilde{\Phi}_{\gamma_{2}(t)}\right|}\right|^{\alpha-\operatorname{avg}\left(\sigma_{\beta_{0}}\right)} \\
& \leq\left|\inf _{t}\left(h^{\beta_{0}}\right)^{\log _{\delta\left(\gamma_{2}(t)\right)}\left|D_{N} \widetilde{\Phi}_{\gamma_{2}(t)}\right|}\right|^{\alpha-\operatorname{avg}\left(\sigma_{\beta_{0}}\right)},
\end{aligned}
$$

since $\delta\left(\gamma_{2}(t)\right) \geq h^{\beta_{0}}$,

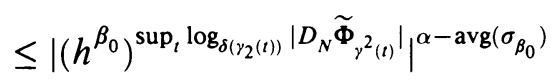

since $\alpha-\operatorname{avg}\left(\sigma_{\beta_{0}}\right)<0$. By definition, this last is

$$
\left|h^{\beta_{0} \lambda_{o}(\rho)}\right|^{\alpha-\operatorname{avg} \sigma_{\beta_{0}}},
$$

where $\rho(t) \equiv \gamma_{h^{\beta_{0}}}(t)$. We conclude that

$$
\left|f\left(\gamma_{2}(h)\right)-f\left(\gamma_{2}(0)\right)\right| \leq|h|^{1+\alpha \beta_{0} \lambda_{0}(\rho)-\beta_{0} \lambda_{0}(\rho) \cdot \operatorname{avg}\left(\sigma_{\beta_{0}}\right)}
$$

which by the choice of $\beta_{0}$ is

$$
\begin{aligned}
& \leq|h|^{\alpha \beta_{0} \lambda_{0}+\beta_{0}\left(1-\lambda_{0}\right)} \\
& =\left|h^{\beta_{0}}\right|^{\alpha \lambda_{0}+\left(1-\lambda_{0}\right)} .
\end{aligned}
$$

Next,

$$
\mathrm{I}=\left|f\left(\gamma_{1}(0)\right)-f\left(\gamma_{1}\left(h^{\beta_{0}}\right)\right)\right| \leq \int_{0}^{h^{\beta_{0}}}\left|\frac{d}{d t}\left(f \circ \gamma_{1}(t)\right)\right| d t .
$$

We estimate the integrand pointwise:

$$
\begin{aligned}
\left|\frac{d}{d t}\left(f \circ \gamma_{1}(t)\right)\right| & =\left|\nabla f\left(\gamma_{1}(t)\right) \cdot \gamma_{1}^{\prime}(t)\right| \\
& =\left|\nabla f\left(\gamma_{1}(t)\right) \cdot \nabla \widetilde{\Phi}_{\gamma_{1}(t)}(0) \cdot \nabla \widetilde{\Phi}_{\gamma_{1}(t)}(0)^{-1} \cdot \gamma_{1}^{\prime}(t)\right|
\end{aligned}
$$

which, as in the estimation if II, is

$$
\leq C\left|D_{N} \widetilde{\Phi}_{\gamma_{1}(t)}\right|^{\alpha} \cdot\left|D_{T} \widetilde{\Phi}_{\gamma_{1}(t)}\right|^{-1} .
$$


But $\gamma_{1}$ is a normal curve so $D_{N}=D_{T}$ and we have

$$
\left|\frac{d}{d t}\left(f \circ \gamma_{1}(t)\right)\right| \leq C\left|D_{N} \widetilde{\Phi}_{\gamma_{1}(t)}\right|^{\alpha-1} \text {. }
$$

Thus

$$
\begin{aligned}
& \mathbf{I} \leq C \int_{0}^{h^{\beta_{0}}}\left|D_{N}\left(\widetilde{\boldsymbol{\Phi}}_{\gamma_{1}(t)}\right)\right|^{\alpha-1} d t
\end{aligned}
$$

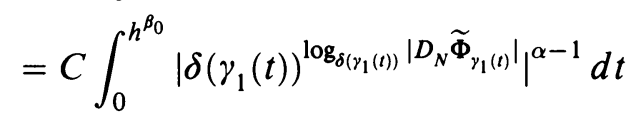

$$
\begin{aligned}
& =C \int_{0}^{h^{\beta_{0}}}|t|^{\left(\log _{\left.\gamma_{1}(t)\right)}\left|D_{N} \widetilde{\Phi}_{\gamma_{1}(t)}\right|\right)(\alpha-1)} d t,
\end{aligned}
$$

by the definition of $\gamma_{1}$. Now, by Lemma 2 , this last is

$$
\begin{aligned}
& \leq C \int_{0}^{h^{\beta_{0}}}|t|^{\left(\log _{\delta\left(y_{1}(0)\right)}\left|D_{N} \widetilde{\Phi}_{\gamma_{1}(0)}\right|\right)(\alpha-1)} \\
& \leq C \int_{0}^{h^{\beta_{0}}}|t|^{\lambda_{0}(\gamma) \cdot(\alpha-1)} d t \\
& =C\left|h^{\beta_{0}}\right|^{\lambda_{0}(\gamma)(\alpha-1)+1} .
\end{aligned}
$$

The estimate of III is similar.

Combining the estimates of I, II, III gives the result for $\alpha$ small, i.e., $\lambda_{0}(\gamma)(\alpha-1)+1<1$.

For a large $\alpha$, higher-order differences are required to get sharp estimates (see [KR4] for the role of higher differences in the study of Lipschitz spaces). A detailed treatment of this case, at least for second-order differences, is in [KR3] For completeness here we outline the idea.

If

$$
1 \leq \lambda_{0}(\gamma)(\alpha-1)+1<2
$$

then Lipschitz smoothness must be measured using $\Delta_{h}^{2}$ (see [KR4]). We define

$$
\begin{array}{ll}
\gamma_{1}(t)=\gamma(0)-t \nu(\gamma(0)), & 0 \leq t \leq h^{\beta_{0}} ; \\
\gamma_{2}(t)=\gamma(2 h)-t \nu(\gamma(2 h)), & 0 \leq t \leq h^{\beta_{0}} ; \\
\gamma_{3}(t)=\gamma(4 h)-t \nu(\gamma(4 h)), & 0 \leq t \leq h^{\beta_{0}} ; \\
\gamma_{4}(t)=\gamma(0)-h^{\beta_{0}} \nu(\gamma(t)), & 0 \leq t \leq h ;
\end{array}
$$

and write

$$
\begin{aligned}
\left|\Delta_{h}^{2} f(\gamma(2 h))\right| \leq & \left|f\left(\gamma_{1}(0)\right)-f\left(\gamma_{1}\left(h^{\beta_{0}}\right)\right)\right|+\left|f\left(\gamma_{2}(0)\right)-f\left(\gamma_{2}\left(h^{\beta_{0}}\right)\right)\right| \\
& +\left|f\left(\gamma_{3}(0)\right)-f\left(\gamma_{3}\left(h^{\beta_{0}}\right)\right)\right|+\left|\Delta_{h}^{2} f\left(\gamma_{4}(2 h)\right)\right| \\
\equiv & \mathrm{I}+\mathrm{II}+\mathrm{III}+\mathrm{IV} .
\end{aligned}
$$


(Note that we evaluate $\Delta_{h}^{2} f(\gamma(\cdot))$ at $2 h$ rather than 0 so that it is well defined; this is purely a notational matter.)

Terms I, II, and III are estimated just as we estimated I, III before. To get a favorable estimate for IV we write

$$
\Delta_{h}^{2} f\left(\gamma_{2}(2 h)\right)=\int_{0}^{2 h} \int_{0}^{2 h}\left(f \circ \gamma_{2}\right)(t+s) d s d t
$$

and estimate the integrand by

$$
\begin{aligned}
\left|\left(f \circ \gamma_{2}\right)^{\prime \prime}(t)\right| & =\left|\left(\left(f \circ \gamma_{2}\right)^{\prime}\right)^{\prime}(t)\right| \\
& =\left|\left(\nabla f \circ \nabla \widetilde{\Phi}_{\gamma_{2}(t)} \circ\left(\nabla \widetilde{\Phi}_{\gamma_{2}(t)}\right)^{-1} \circ \gamma_{2}^{\prime}\right)^{\prime}\right| \\
& =\left|\left(\nabla^{2}\left(f \circ \widetilde{\Phi}_{\gamma_{2}(t)}\right)\right) \circ\left(\left(\nabla \widetilde{\Phi}_{\gamma_{2}(t)}\right)^{-1} \circ \gamma_{2}^{\prime}\right)^{\prime}\right| \\
& \leq C \cdot\left|D_{N} \widetilde{\Phi}_{\gamma_{2}(t)}\right|^{\alpha} \cdot\left|D_{T} \widetilde{\Phi}_{\gamma_{2}(t)}\right|^{-2} .
\end{aligned}
$$

Now, proceeding as in the argument with the first difference, we obtain a favorable estimate. Higher-order differences are handled similarly.

\section{More on the Kugelsatz Case}

As mentioned in $\S 2$, the correct result for a domain of the form

$$
\Omega=B(0,2) \backslash \overline{B(\mathbf{1}, 1)}
$$

near the point $\mathbf{1} \equiv(1,0) \in \partial \Omega$ is that a holomorphic $\Lambda_{\alpha}(\Omega)$ function is in $C^{\infty}(\overline{\Omega \cap B(\mathbf{1}, 1)})$. However, the machinery set up to prove our main theorem does not yield this result. Here we show how to modify the argument to get the full result.

Let $z \in \Omega,|z-\mathbf{1}|<1$, and consider the curve $\gamma(t)=z-t \nu(z), 0 \leq$ $t \leq 1$. We will prove that if $f$ is holomorphic and $\Lambda_{\alpha}$ in $\Omega \cap B(1,1)$, any $0<\alpha<\infty$, then $f \circ \gamma$ is $\Lambda_{\alpha+1 / 3-\varepsilon}$, any $\varepsilon>0$. By the result of [KR5], it follows that $f \in \Lambda_{\alpha+1 / 3-\varepsilon}(\overline{\Omega \cap B(1,1)})$. Iteration of this argument shows that $f \in \Lambda_{\beta}(\overline{\Omega \cap B(\mathbf{1}, 1)})$ for all $\beta>0$. Hence $f \in C^{\infty}(\overline{\mathbf{\Omega} \cap B(\mathbf{1}, 1)})$.

Let $\alpha+1 / 3<k \in \mathbf{Z}$. For $h>0$ small and $t_{0}$ sufficiently large $\left(t_{0}>2 k h\right)$ we estimate

$$
\Delta_{h}^{k} f\left(\gamma\left(t_{0}\right)\right) \text {. }
$$

Letting $\beta_{0}=4 k /(3 k+1)$, we define as usual

$$
\begin{aligned}
\gamma^{1}(t) & =\gamma\left(t_{0}-k h\right)-t \nu\left(\gamma\left(t_{0}-k h\right)\right), \\
\gamma^{2}(t) & =\gamma\left(t_{0}-(k-1) h\right)-t \nu\left(\gamma\left(t_{0}-(k-1) h\right)\right), \\
\vdots & \\
\gamma^{2 k-2}(t) & =\gamma\left(t_{0}+(k-1) h\right)-t \nu\left(\gamma\left(t_{0}+(k-1) h\right)\right), \\
\gamma^{2 k-1}(t) & =\gamma\left(t_{0}+k h\right)-t \nu\left(\gamma\left(t_{0}+k h\right)\right), \\
\gamma^{2 k}(t) & =\gamma(t)-h^{\beta_{0}} \nu(\gamma(t)) .
\end{aligned}
$$


Then, as usual,

$$
\begin{aligned}
\mid \Delta_{h}^{k} f\left(\gamma\left(t_{0}\right) \mid\right. & \leq \sum_{j=1}^{2 k-1}\left|f\left(\gamma^{j}\left(h^{\beta_{0}}\right)\right)-f\left(\gamma^{j}(0)\right)\right|+\left|\Delta_{h}^{k} f\left(\gamma^{2 k}\left(t_{0}\right)\right)\right| \\
& \equiv T_{1}+T_{2}+\cdots+T_{2 k} .
\end{aligned}
$$

Now we estimate $T_{1}, \ldots, T_{2 k-1}$ just as was done for I, II, III in the treatment of second differences at the end of the last section. The term $T_{2 k}$ is estimated by writing

$$
\Delta_{h}^{k} f\left(\gamma^{2 k}\left(t_{0}\right)\right)=\int_{0}^{2 h} \int_{0}^{2 h} \cdots \int_{0}^{2 h}\left(f \circ \gamma^{2 k}\right)^{(k)}\left(t_{0}-2 k h+s_{1}+\cdots+s_{k}\right) d s_{1} \cdots d s_{k}
$$

and proceeding as before. The result is that

$$
\left|\Delta_{h}^{k} f\left(\gamma\left(t_{0}\right)\right)\right| \leq C|h|^{k(3 \alpha+1) /(3 k+1)} .
$$

We see that if $k$ is sufficiently large this yields (see [KR4]) that $f \circ \gamma \in \Lambda_{\alpha+1 / 3-\varepsilon}$ for any $\varepsilon>0$. It follows that $f \circ \gamma \in C^{\infty}\left[0, h_{0}\right]$. By results of [KR5], $f \in C^{\infty}(\overline{\Omega \cap B(0,1)})$. This completes the proof.

\section{REFERENCES}

[CA] D. Catlin, Estimates of invariant metrics on pseudoconvex domains of dimension two, preprint.

[CK] Der Chen E. Chang and S. G. Krantz, Holomorphic Lipschitz functions and applications to the $\bar{\partial}$-problem, preprint.

[GR] I. Graham, Boundary behavior of the Caratheodory and Kobayashi metrics on strongly pseudoconvex domains in $\mathbf{C}^{n}$ with smooth boundary, Trans. Amer. Math. Soc. 207 (1975), 219-240.

[GK] R. E. Greene and S. G. Krantz, Stability of the Carathéodory and Kobayashi metrics and applications to biholomorphic mappings, Proc. Sympos. Pure Math., vol. 41, Amer. Math. Soc., Providence, R. I., 1984, pp. 77-93.

[GRR] H. Grauert and H. Reckziegel, Hermitesche Metriken und narmale Familien holomorpher Abbildungen, Math. Z. 89 (1965), 108-125.

[KR1] S. G. Krantz, Fatou theorems on domains in $\mathbf{C}^{n}$, Bull. Amer. Math. Soc., 16 (1987), 93-96.

[KR2] _ Invariant metrics and boundary behavior of holomorphic functions, in preparation.

[KR3] _ Function theory of several complex variables, Wiley, New York, 1982.

[KR4] _ Lipschitz spaces, smoothness of functions, and approximation theory, Expositiones Math. 3 (1983), 193-260.

[KR5] _ Smoothness of harmonic and holomorphic functions, Proc. Sympos. Pure Math., vol. 35, Amer. Math. Soc., Providence, R. I., 1979, pp. 63-67.

[KR6] _ Boundary values and estimates for holomorphic functions of several complex variables, Duke Math. J. 47 (1980), 81-98.

[KR7] _ The boundary behavior of the Kobayashi metric, Rocky Mountain J. Math. (to appear).

[KM] S. G. Krantz and Daowei Ma, Bloch functions on strongly pseudoconvex domains, Indiana Math. J. (in press).

[RE] H. Reiffen, Die differentialgeometrischen Eigenschaften der invarianten Distanzfunktion von Carathéodory, Schr. Math. Inst. Univ. Münster 26 (1963). 
[RO] H. Royden, Remarks on the Kobayashi metric, Lecture Notes in Math., vol. 185, SpringerVerlag, Berlin, 1971.

[ST] E. M. Stein, Singular integrals and estimates for the Cauchy-Riemann equations, Bull. Amer. Math. Soc. 79 (1973), 440-445.

Department of Mathematics, Washington University, St. Louis, Missouri 63130 